\title{
edmetic
}

Revista de Educación Mediática y TIC

La competencia digital de los políticos españoles en Twitter. El caso de las elecciones europeas de 2014

The digital competence of Spanish politicians in Twitter. The case of the European Elections 2014

Fecha de recepción: 29/06/2017

Fecha de revisión: 12/06/2017

Fecha de aceptación: 14/06/2017 


\title{
La competencia digital de los políticos españoles en Twitter. El caso de las elecciones europeas de 2014 The digital competence of Spanish politicians in Twitter. The case of the
}

\section{European Elections 2014}

\section{Roberto Gelado Marcos ${ }^{1}$ y Fernando Bonete Vizcaíno²}

\begin{abstract}
Resumen:
Twitter se ha convertido en un controvertido objeto de debate en varios aspectos de la vida pública. Sin embargo, antes de plantearnos la educación mediática que eclosiones como la de Twitter requiere de una ciudadanía que ve la oportunidad de sumergirse de nuevo en profundidad en la esfera pública, cabe preguntarse si actores de suma importancia en ella como los políticos entienden bien estas nuevas posibilidades de comunicación con sus gobernados. Este artículo pretende contribuir al debate entre quienes defienden el potencial de la red de microblogging y quienes constatan, en cambio, que los actores que la utilizan no están exprimiendo tal potencial. Para ello, hemos analizado el uso que de Twitter se hizo por parte de los líderes políticos españoles más significativos durante las Elecciones Europeas de 2014, combinando a tal fin metodologías cuantitativas y cualitativas que permitan un análisis más profundo de sus discursos y su comprensión de la herramienta.
\end{abstract}

Palabras claves: Competencia comunicativa, Iniciación informática, España, Medios de comunicación de masas

\begin{abstract}
:
Twitter has become a controversial topic for debate in numerous aspects of the public life. However, before asking ourselves what kind of media education booms like Twitter require from a citizenship that can immerse again in the public sphere, we may wonder whether highly important figures in the configuration of this public sphere -politicians, for instance- do understand the new possibilities that technologies enable to enhance the communication with the people they rule. This paper aims at contributing to the debate between those who defend the potential of this microblogging tool, and those who prove with their studies that those actors that are using it are not making the most of this potential. In order to do so, we have analysed how Twitter was used by Spanish political leaders during the European Elections of 2014. Quantitative and qualitative methodologies will be used to fully analyse their discourses.
\end{abstract}

Keywords: Political Socialization, Communicative competence, Computer literacy, Mass media

\footnotetext{
1 Universidad CEU San Pablo, Madrid (España); roberto.geladomarcos@ceu.es

2 Universidad CEU San Pablo, Madrid (España); fernando.bonetevizcaino@ceu.es
} 


\section{Introducción}

Twitter se ha venido definiendo históricamente como una red social conformadora de grandes corrientes y flujos de opinión que marcan tendencia. La herramienta se ha entendido siempre, o al menos sus creadores parecen verla así, como el resultado de mensajes emitidos por usuarios que interactúan entre sí a un mismo nivel, para expresar inquietudes cotidianas y, en general, estimular el debate en múltiples esferas. Este potencial de comunicación directa, sin intermediarios no ha tardado en atraer la atención de los profesionales de la comunicación política. La presencia de partidos políticos y sus líderes en Twitter se ha convertido en pocos años en un punto de paso inexcusable en la hoja de ruta de la nueva política, que pretende, entre otras cosas, trasladar una sensación mayor de cercanía del político a los ciudadanos; en un intento, probablemente, de por paliar la creciente desafección hacia la clase política. Así, Twitter parece revelarse como la herramienta perfecta para que el político se presente a la ciudadanía como un usuario más que comparte criterios y discute pareceres acerca de los

grandes temas de debate público, lo cual podría traer aparejado, además, el mucho más estimulante efecto de revitalizar la democracia a través de una nueva participación pública.

Sin embargo, más allá del indudable éxito, la creciente presencia de formaciones políticas en Twitter, y el innegable uso que los militantes hacen de esta red de microblogging, no abundan precisamente las respuestas cuando comienzan a proliferar preguntas sobre cómo emplearla de manera efectiva para conseguir los objetivos de comunicación deseados. Hacer política en esta red social, con herramientas y posibilidades tan distintas a las brindadas por los medios de comunicación tradicionales, y más próxima a la Red (Bernal y Congosto, 2014) supone, sin duda, un reto en muchos sentidos.

Siguiendo el excelente análisis de la política estadounidense de Parmelee and Bichard (2011), se asentó, entre profesionales y académicos, la idea optimista de que Twitter iba a introducir un cambio radical en los hábitos comunicativos de la esfera política, como probaba el hecho de que fuera ya una herramienta indispensable para los líderes políticos y la articulación de sus 
campañas electorales. Desde luego que el potencial de esta red social para fomentar el modelo de democracia participativa, ofreciendo a los usuarios la oportunidad de influenciar en la agenda temática a través de sus mensajes y el uso masivo de hashtags, e incluso provocar el cambio, es innegable a escala internacional (Shirky, 2011), como no es menos evidente la magnífica oportunidad brindada a los políticos para que aprovechen este nuevo modelo participativo, ya sea aportando su perspectiva en los muchos frentes abiertos en el debate público o, al menos, como herramienta de medición de los temas que importan a los ciudadanos (Tumasjan, Sprenger, Sandner y Welpe, 2010). En la inquietud de discernir hasta qué punto los principales líderes políticos españoles aprovecharon esta oportunidad en las pasadas Elecciones al Parlamento Europeo, celebradas en 2014, y en qué grado, reside la principal preocupación de este estudio.

La política española ha dado dos grandes ocasiones previas a las Elecciones Europeas de 2014 para el análisis, la primera de las cuales tuvo lugar en 2011 , año de convocatoria de las Elecciones Generales, dando como resultado los primeros análisis del fenómeno. Cebrián y Vázquez (2013) se centraron entonces en el uso de Twitter por los dos principales candidatos a la presidencia: el líder del Partido Popular (PP) Mariano Rajoy y el del Partido Socialista Obrero Español (PSOE) Alfredo Pérez Rubalcaba. Este trabajo dejó al descubierto la falta de correspondencia entre las acciones llevadas a cabo por ambos candidatos y la participación ciudadana; una conclusión igualmente confirmada por el análogo análisis de Zurutuza (2012), para quien Twitter se utilizaba entonces al estilo de los canales de comunicación tradicional, como una herramienta de difusión de propuestas políticas e ideológicas, sin intenciones expresas de interactuar con los ciudadanos y abrir nuevas vías de diálogo que pudieran enriquecer el debate público.

Un segundo momento lo protagonizan las elecciones de 2012 celebradas en distintas comunidades autónomas: Andalucía, Cataluña, Galicia y País Vasco. Deltell, Claes y Osteso (2013), de un lado, y Cebrián, Vázquez y Olabarrieta (2013), de otro se centraron en los casos andaluz y vasco y, tras analizar la correlación entre los mensajes emitidos por partidos y líderes políticos, y la intencionalidad del debate abierto por los ciudadanos, 
llegaron a conclusiones similares a las anteriormente apuntadas: ni hubo intención de establecer un debate público mínimamente original y relevante, ni existió un diálogo entre candidatos y electores propio y efectivo.

Con estos antecedentes como telón de fondo, es evidente una cierta polarización académica entre el optimismo de quienes subrayan el potencial dinamizador de Twitter en la esfera política y quienes constatan, tras analizar diversos momentos electorales, que este potencial no se plasma en una realidad interactiva. Es por ello que las Elecciones al Parlamento Europeo de 2014 devienen en un interesante caso de estudio cuyo análisis puede contribuir a arrojar algo más de luz a este encendido debate. Las Elecciones al Parlamento Europeo de 2014 dotaron al análisis de estas realidades de una nueva dimensión internacional colectiva y una oportunidad investigadora más segura que las previas de 2009, donde Web, aplicaciones móviles y medios sociales empezaban entonces a hibridarse con la política y su éxito y conveniencia era todavía inciertos (Michailidou y Trenz, 2010; Vergeer, Hermans y Cunha, 2012). Pertenecen al ámbito de estas investigaciones pioneras en torno a Twitter y su influencia en las elecciones numerosos trabajos de índole internacional referidos al territorio europeo, que vendrían a conformar una primera aproximación a las cuestiones que luego planteará la ocasión brindada en 2014 . Algunos de los más representativos, procedentes de diferentes países: en Rumanía, Holotescu, Gutu, Grosseck, Bran (2009); desde Suecia y Dinamarca, Larson y Moe $(2011$, 2013); en Alemania, Mambrey y Dörr (2011); en Holanda, Vergeer y Hermans (2013); en Grecia, Ferra y Karatzogianni (2018); en Reino Unido, Houghton, Hamdan y Marder (2017); y, desde una perspectiva comparativa entre Reino Unido, Francia y Estados Unidos, Lilleker y Jackson (2013).

La práctica totalidad de estos trabajos explotaban todavía el término "microblogging", sin centrar todavía su atención en Twitter, diversificando sus esfuerzos entre las redes sociales disponibles en el momento.

De vuelta al caso español, y de nuevo a las Elecciones Europeas de 2014, el surgimiento de nuevos partidos y, en concreto, el rápido ascenso de Podemos en la escena política española, reforzado por el contundente éxito 
de la coalición de izquierda griega SYRIZA (The Economist, 2014), confirieron una supuesta novedad al fenómeno, que contaría a partir de entonces con una formación política joven e inquieta, en principio familiarizada con el entorno del social media e incluso, según diversos medios de comunicación, auténticamente creativa en su uso diario (Bollero, 2014). Sin embargo, las primeras aproximaciones referidas uso de Twitter durante el transcurso de la campaña, primero en forma de comparativa PP-Podemos (Zugasti y Pérez, 2015), más tarde concretando en la figura del líder del último partido, Pablo Iglesias (Bonete, Cebrián y Vázquez, 2016), terminaron por declarar la ausencia de un auténtico diálogo de los partidos y sus líderes con los ciudadanos.

Zanjar esta cuestión completando la investigación mediante el análisis comparado de los tuits lanzados por los líderes de los partidos más votados nos permite comenzar a reunir respuestas sólidas referentes a interrogantes de la máxima importancia en torno al uso que los políticos españoles han hecho hasta el momento de Twitter.

\subsection{Las Elecciones Europeas}

La aparición de los nuevos medios de comunicación social no ha supuesto el único cambio reseñable de esta última etapa de los comicios europeos. La propia configuración de la escena política española ha experimentado fuertes transformaciones en los cuatro años previos a la celebración de las últimas elecciones al Parlamento Europeo. Si en 2009 apenas era posible determinar cuál sería el resultado final en la evolución y uso de las nuevas plataformas sociales en el contexto de la política, tampoco lo era pensar que la tradición bipartidista, según la cual Partido Popular (PP) y Partido Socialista Obrero Español (POSE) habían absolutizado la representación política tanto en España como en Europa, podría llegar a resquebrajarse para dar lugar a la viva presencia y éxito de nuevos partidos de muy reciente creación.

Si nos remontamos a 2004 y realizamos una rápida comparativa con los resultados de 2009 obtenidos en las elecciones al Parlamento Europeo, comprobaremos que, de los 54 escaños asignados a España, PP y PSOE nunca sumaron juntos menos de 47 escaños: 24 escaños para el PP en ambas ocasiones y 25 y 23 para el PSOE, respectivamente. Otras formaciones políticas 
apenas aglutinaban una representación reseñable en el conjunto de los votos, siquiera mediante grandes coaliciones. En ambas citas electorales repitieron resultados: Europa de los Pueblos (EdP), con la obtención de 1 escaño; la izquierda de Iniciativa per Catalunya Verds, Esquerra Unida i Alternativa e Izquierda Unida, juntos con 2 escaños (en 2009 se sumó Bloque por Asturies); o la triple alianza de Galeusca (en 2009 bajo el nombre de Coalición por Europa), con 3 escaños. Ni en 2004 ni en 2009 ninguna de las coaliciones se acercaron al millón de votos, si bien en aquella última ocasión un partido, Unión Progreso y Democracia (UPyD), presentado como primera formación contra el bipartidismo, logró él solo 1 escaño con casi medio millón de votos. El discreto éxito de UPyD supuso el primer desafío a la hegemonía bipartidista del PP y el PSOE.

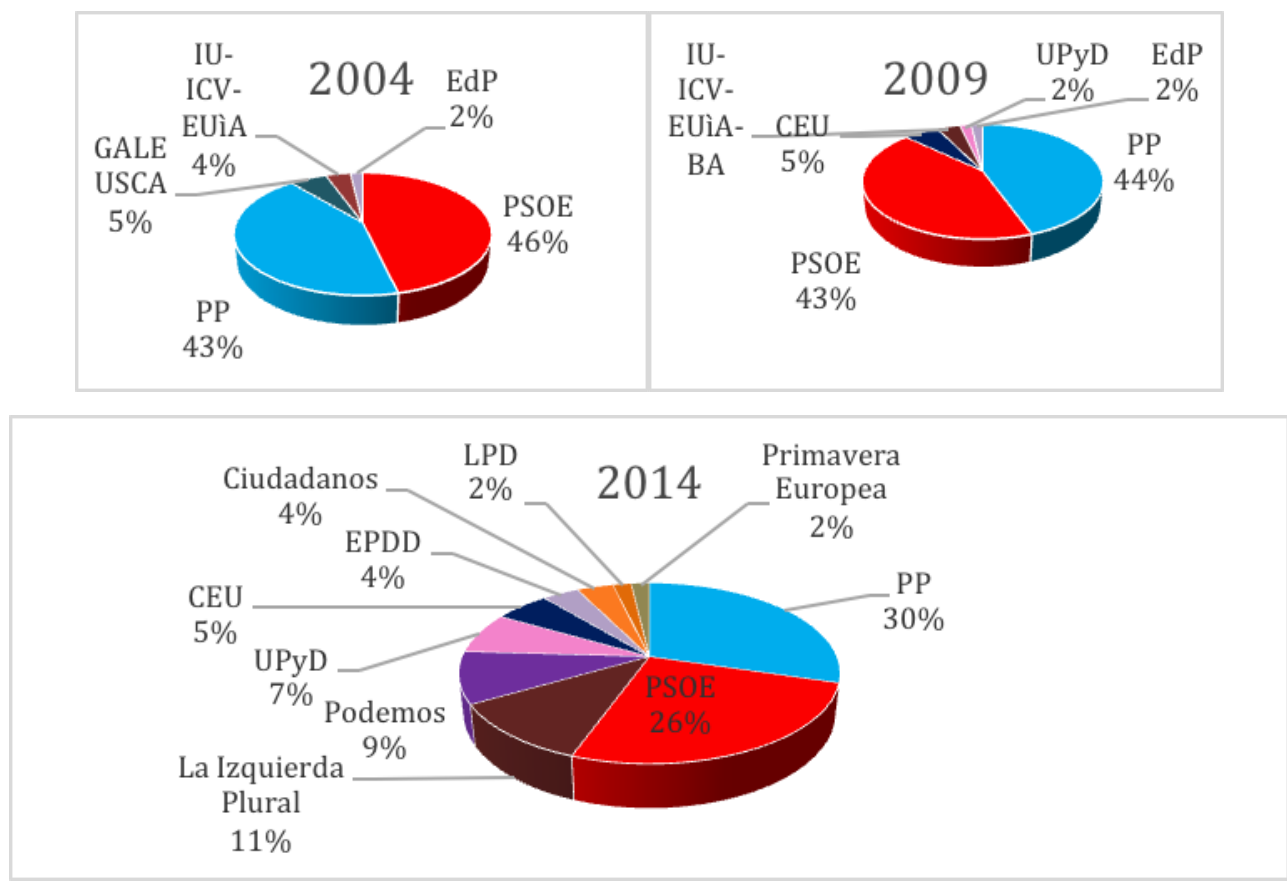

Gráfico 1. Comparativa histórica de resultados de las Elecciones Europeas en España. Fuente: Parlamento Europeo

Desde entonces, la escena política española cambiaría por completo. El descontento social dirigido hacia los dos partidos mayoritarios por la gestión realizada durante la crisis económica y los escándalos de corrupción interna, y las cada vez más numerosas peticiones de cambio y reforma constitucional, 
favorecieron la atracción del voto por parte de los partidos hasta entonces minoritarios, y dispusieron la llegada de nuevas formaciones políticas de corte mucho más radical. El total de 47 escaños obtenidos por PP y PSOE en las elecciones de 2009, se vio reducido hasta los 30 escaños de 2014. A las coaliciones habituales, que mantuvieron sus escaños o los aumentaron, cabe añadir la presencia de nuevos partidos, que como Podemos (5 escaños), Ciudadanos (2 escaños), o las coaliciones L'Esquerra pel Dret a Decidir (EPDD, 2 escaños) Los Pueblos Deciden (LPD, 1 escaño) y Primavera Europea (1 escaño) incurrieron por primera vez en unas elecciones y lograron representación política en Europa.

Además, por vez primera en una Elecciones Europeas en España, formaciones políticas diferentes a las dos opciones mayoritarias conformadas por el PP y el PSOE, como la coalición de izquierdas La Izquierda Plural, y los partidos Podemos y UPyD, lograron llegar y superar el millón de votos.

\section{Metodología}

La propia definición de Twitter como una red social conformadora de grandes corrientes y flujos de opinión que marcan tendencia, resultado de los mensajes emitidos por usuarios que interactúan entre sí a un mismo nivel, que expresan inquietudes cotidianas y permiten conectar personas de manera directa, sin intermediarios, ha hecho del espacio creado por esta herramienta el entorno perfecto para la irrupción de la comunicación política. La presencia de partidos políticos y sus líderes en Twitter se ha convertido ya en un requerimiento obligado para poder seguir el nuevo ritmo de la política, marcado por el supuesto acercamiento del político a los ciudadanos, como un usuario más que comparte criterios y discute pareceres acerca de los grandes temas de debate público, revitalizando de ese modo la democracia a través de una nueva participación pública.

Cuatro son las preguntas fundamentales que han articulado nuestra investigación y de las que nos hemos servido para desentrañar el uso que los líderes de los principales partidos políticos españoles hicieron de Twitter durante las Elecciones Europeas de 2014. Las presentamos a continuación: 
1. ¿Están familiarizados los líderes políticos españoles con Twitter como herramienta de comunicación?

2. ¿̇Es Twitter para los líderes políticos una herramienta de interacción e información o un medio para la propaganda y la autopromoción?

a. Si prevalece la autopromoción, ¿̇se centra en la propia figura del líder político o se articula en un sentido más laxo, como medio para hacer referencia al partido en general o a sus compañeros de filas?

3. ¿Cuál es la naturaleza del discurso político utilizado? ¿Qué temas abordan y cual es el lenguaje político utilizado?

4. ¿̇Enriquece Twitter el debate político en España proponiendo, abordando y discutiendo temas de interés público y preocupaciones ciudadanas?

El marco temporal establecido por la investigación cubre el antes y el después de la jornada electoral del 25 de mayo de 2014: las tres semanas previas y una semana posterior, del 4 de mayo al 1 de junio. En cuanto al objeto de estudio, se acotaron tanto el número de partidos que formarían parte del análisis como su naturaleza e identidad.

La cantidad de cuentas integradas en el análisis estuvo determinada por la representación obtenida por cada uno de los partidos concurrentes a las elecciones: formaron parte de la muestra aquellos partidos que lograron aglutinar más de un millón de votos, es decir, aquellos con una representatividad suficientemente significativa.

Con respecto a la decisión de qué perfil de Twitter representaría a cada uno de esos partidos, se tuvo en cuenta la personalización con que se identifica tradicionalmente a la política española (Bosco, 2013; Albaek, Van Dalen, Jebril y De Vreese, 2014), para elegir las cuentas de los candidatos, con la única excepción de UPyD, cuyo candidato, Ricardo Sosa-Wagner, no poseía cuenta en la red social en el momento de la celebración de la campaña electoral. En este caso, se procedió a incluir en su lugar la cuenta que el partido habilitó para asuntos europeos. Entre los mensajes de estas cuentas que formaron parte del análisis, y teniendo en cuenta que el objetivo 
principal de esta investigación se centra en desvelar la naturaleza del discurso político de estos líderes en Twitter, se primaron los tuits emitidos, dejando a un lado las respuestas.

Con todo, la muestra final está formada por las cuentas de Twitter de los candidatos del Partido Popular (Miguel Arias Cañete: @MAC_europa), Partido Socialista Obrero Español (Elena Valenciano: @ElenaValenciano), La Izquierda Plural (Willy Meyer: @willymeyerlu), Podemos (Pablo Iglesias: @Pablo_Iglesias_), y UPyD (@UPyDEuropa).

Los tuits de estas cuentas para el marco temporal fijado más arriba fueron recuperados mediante el programa informático Nvivo 10. El uso de esta herramienta nos permitió manejar una extensa muestra final conformada por el total de unos 3.000 tuits por cuenta y facilitar el exhaustivo análisis de carácter cuantitativo y cualitativo necesario para el objetivo fijado por la investigación.

\subsection{Diseño de la investigación}

Una vez establecidos los interrogantes origen de la investigación, los criterios marco para el estudio y las herramientas para acometerlo, el análisis tuvo un recorrido en dos etapas. En primer lugar, una aproximación lingüística al discurso elaborado por cada uno de los líderes políticos gracias a la herramienta de búsqueda y frecuencia de palabras clave suministrada por Nvivo. Este proceso ofreció las tendencias clave de cada uno de los discursos y, además, la oportunidad de establecer paralelismos y puntos de divergencia entre ellos.

En segundo lugar, de este estadio cuantitativo, y en consonancia con los objetivos de análisis cualitativo proyectados por la investigación, se siguió un estudio en profundidad para desvelar la verdadera naturaleza y finalidad de los mensaje lanzados y del discurso en su totalidad, que tuviera en cuenta todas sus implicaciones y significado en la escena política del momento (Gee, 1999).

Con todo, el resultado de la investigación se articula en torno a un modelo integrado por cuatro categorías clave compartidas, válidas, para todas las cuentas estudiadas: (1) "Menciones", en las que se incluyen también 
las (1.1) "Automenciones", (1.2) "Menciones a otros" y (1.3) "Hashtag"; (2) "Campaña electoral"; (3) "Lenguaje político"; y (4) "Temas políticos".

\section{Resultados}

Tal y como se ha reseñado con anterioridad, si bien el diseño de la investigación tiene como última finalidad desembocar en un estudio cualitativo preciso del discurso político desplegado por los líderes políticos españoles tanto en la campaña electoral previa a las Elecciones y durante los días posteriores a la cita electoral, una primera aproximación a su actividad en Twitter en ese marco temporal nos proporciona un valioso punto de partida para establecer su grado de conocimiento de las estrategias que constituyen la denominada comunicación 2.0, antes de valorar su uso.

\subsection{Política y Europeas en Twitter. Una mirada cuantitativa.}

Un primer vistazo al papel desempeñado por Twitter a la hora de conformar la estrategia y discurso político de los candidatos españoles durante y tras el periodo electoral revela claras diferencias en cuanto a la importancia que cada uno de los líderes asignó a esta plataforma. Uno de los casos más sorprendentes fue precisamente el del partido que de hecho ganaría las elecciones: el Partido Popular, a través de su candidato Miguel Arias Cañete, que estrenó cuenta de Twitter justo al inicio de la campaña y su producción fue verdaderamente escasa en comparación con sus rivales.

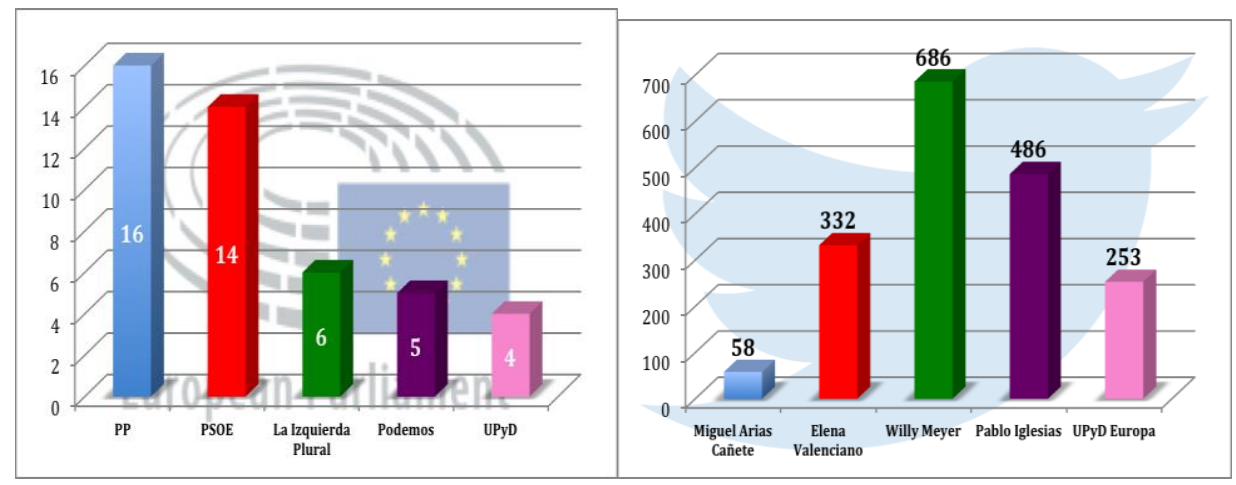

Gráfico 2. Comparativa de resultados electorales (arriba) y producción de tuits (abajo). Fuente: Parlamento Europeo y elaboración propia. 
58 fueron los tuits publicados por Arias Cañete durante el total de cuatro semanas estudiadas, apenas un cuarto de los 253 publicados por la cuenta de UPyD Europa, una sexta parte de los 332 de Elena Valenciano, una octava parte de los 486 de Pablo Iglesias y una doceava parte de los 686 publicados por Willy Meyer (IP). Todo lo cual parece refutar la idea de una significativa correlación entre la frecuencia de actividad en Twitter y el éxito en las urnas, tal y como se muestra en el Gráfico 2.

En cualquier caso, extender la comparación y tener en cuenta los resultados de los comicios celebrados en 2009 podría ofrecer una visión más matizada del asunto. Es claro que el tradicional bipartidismo español ha marcado la política española y sus resultados electorales desde la Transición y el nacimiento de la democracia, y que esta hegemonía de los dos partidos mayoritarios del país se ha visto igualmente reflejada en el ámbito europeo. En 2009, de los 50 escaños en el Parlamento Europeo reservados para España, el PP obtuvo 24 y el PSOE 23, es decir, 47 del total. Solo tres fueron para otros partidos: IP, con 2 escaños, y UPyD, con 1.

Sin embargo, en 2014, pese a mantenerse la bipartición general de los votos y no hallar una correspondencia real entre tuits y votos, lo cierto es que el candidato más prolífico en Twitter y su partido, IP, lograron pasar de los 2 escaños de 2009 a 6 en 2014 y que el también activo Pablo Iglesias y Podemos lograron, en la primera cita electoral de su corta historia política, obtener 5 escaños en el Parlamento Europeo.

No solo la cantidad, también la originalidad de los mensajes emitidos marcó diferencias entre los candidatos estudiados. Para algunos, como se puede comprobar en el Gráfico 3, la cantidad prevaleció sobre la originalidad en su estrategia, antes y después de las elecciones. 


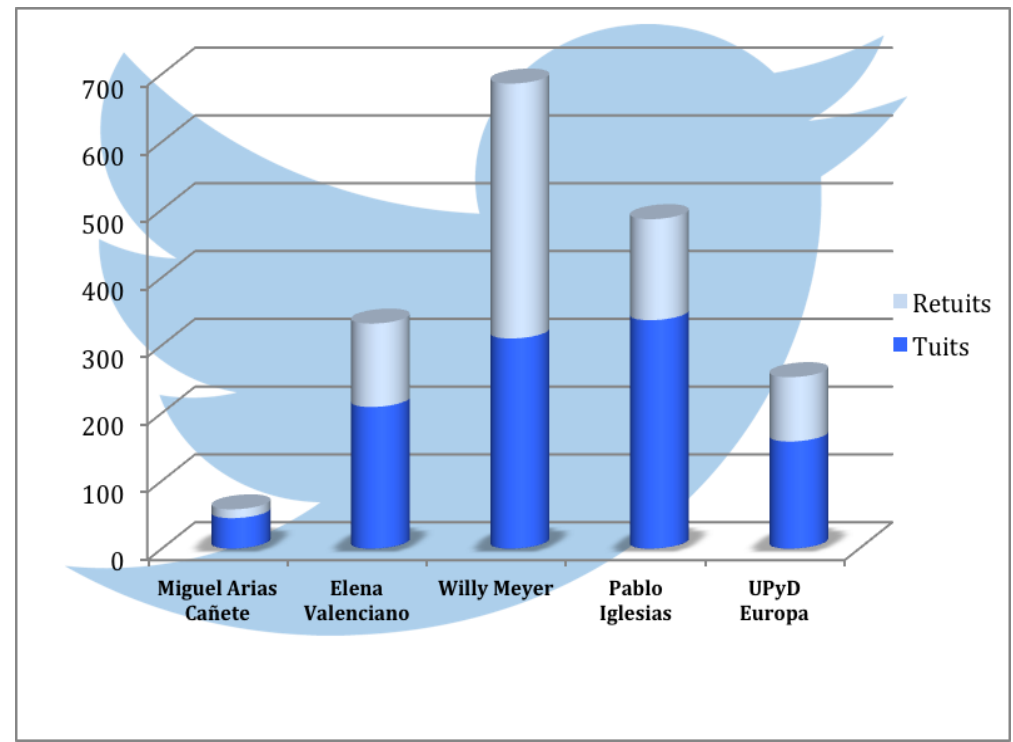

Gráfico 3. Ratio de originalidad: porcentaje de tuits propios sobre número total de tuits publicados. Fuente: Elaboración propia.

Teniendo en consideración este baremo de tuits propios, la cuenta de Pablo Iglesias escala hasta el primer puesto y se convierte en la más prolija, incluso por encima de Willy Meyer, de quien se contabilizó más de un retuit por cada nuevo tuit no publicado con anterioridad, y por tanto su cuenta resultó ser de menor originalidad. Elena Valenciano y UPyD Europa se situán a la zaga de Pablo Iglesias con ratios de originalidad similares. El porcentaje de Arias Cañete es, como se puede suponer, relativo, si tenemos en cuenta la escasez en cuanto al número total de tuits publicados: su ratio de originalidad es de un 77,59\%, pero su producción sigue siendo escasísima: 58 tuits en total.

Examinando el mismo ratio en el resto de cuentas examinadas arroja unos datos no excesivamente alentadores, ya que ninguna de ellas supera el 70\% de originalidad: Pablo Iglesias publica un 69,34\% de tuits propios; Elena Valenciano, un 62,95\%; UPyD Europa, un 62,45\%; y, en el caso de Willy Meyer, ni si quiera la mitad de sus tuits son propios, solo un $45,19 \%$ lo son.

\subsection{Análisis cualitativo del uso de Twitter por parte de los políticos españoles}

Tal y como se mencionó en la metodología expuesta más arriba, una primera aproximación al análisis del discurso fue realizada mediante la herramienta de búsqueda y frecuencia de palabras de Nvivo. Esto proporcionó una 
categorización del análisis a través de cuatro grandes áreas de estudio: "Menciones", "Campaña electoral", "Lenguaje político" y "Temas políticos". Los tuits fueron asignados a estas categorías como parte de una primera clasificación cualitativa más tarde completada con un análisis en profundidad del contenido para detectar y fijar parecidos que fueran más allá de la coincidencia terminológica.

Con todo, similitudes y diferencias en las cláusulas ideológicas expresadas por los políticos en Twitter, fueron identificadas. Al mismo tiempo que se cumplía con este objetivo fundamental de investigación se atendió a valorar el potencial de Twitter como herramienta de estimulación del debate político y mejora de la comunicación e interacción entre candidatos y votantes.

\subsubsection{Miguel Arias Cañete}

Ya se hizo notar con anterioridad la falta de familiaridad del candidato Popular con las formas de comunicación 2.0: su cuenta de Twitter fue activada con la campaña electoral ya en marcha, y la improvisación, materializada en el escaso número de tuits lanzados por el candidato, se hizo notar durante la misma. Tras las Elecciones y el triunfo electoral, expresado en su nombramiento como Comisario Europeo de Acción por el Clima y Energía, su cuenta fue objeto de una cierta reactivación que tuvo como resultado la publicación de 1.500 tuits en ocho meses. Sin embargo, durante el desarrollo de la campaña y en el periodo inmediatamente posterior a las elecciones se vieron marcados por la falta de actividad, con escasos 60 tuits en cuatro semanas.

En este caso de características tan marcadas y evidentes, la aproximación cuantitativa a la cuenta de Arias Cañete desvela ya las claves que la investigación cualitativa no hace sino confirmar: el discurso del candidato popular está conformado en su mayoría por términos y estrategias procedentes de la comunicación política más tradicional, vertidos directamente sobre Twitter y su nuevo contexto interactivo.

De hecho, los hashtags más utilizados por la cuenta se limitan a interpelar ciudadano pidiendo el voto (\#Votacañete, \#votapp), y cualquier otro tipo de hipercontextualización se ve reducida a mencionar al Partido 
Socialista: no se encuentran interacciones con otros candidatos políticos más allá de los rivales tradicionales, ni intentos de iniciar o participar en un verdadero debate político a través de Twitter.

Con todo, hay que añadir que los temas tratados por Arias Cañete son también pocos, y las ocasiones en que presenta otros diferentes al margen de los habituales, como los englobados en etiquetas clave como "Agricultura", "España" y "Europa", se trata más bien de una estratégica reactiva o circunstancial, que de una hoja de ruta previamente planeada. Es el caso de los temas expuestos sobre igualdad de género con anterioridad al debate televisado con Elena Valenciano, de manera aislada para aquella ocasión, y seguramente con la intención de prevenir posibles ataques de la candidata socialista.

\subsubsection{Elena Valenciano}

El discurso proyectado por Elena Valenciano marca la primera diferencia con respecto al de Arias Cañete en su mayor profusión. Además, posee, en líneas generales, un estilo menos combativo que el de sus rivales políticos. Por el contrario, la interacción con otros usuarios fue prácticamente nula, más allá de debates esporádicos con sus rivales políticos tradicionales, como si se quisiera extender el bipartidismo imperante en los últimos años también al contexto social de los nuevos medios.

Su estilo es personal, a veces incluso desenfadado, como en aquellas ocasiones en las que utiliza palabras cortas e incisivas o usa expresiones coloquiales como "meterse a saco" en su tuit "Por una España que se meta a saco en un Plan de Empleo Juvenil"; lo cual puede ser interpretado como un intento de dirigirse a los usuarios más jóvenes y de personalizar el tono de los mensajes dependiendo del grupo objetivo.

El uso de los hashtag crece tanto en cantidad como en coherencia, con casi un tercio de los tuits publicados (109 de 332) gravitando en torno al eslogan de campaña \#TumuevesEuropa: una llamada directa al voto, pero de mayor imaginación y mucho más personal que el elaborado por Arias Cañete. 
También en comparación con el candidato Popular, la variedad de los temas articulados es mayor: "Europa" (76 tuits), "Mujeres" (29), "Políticas sociales" (14), "Igualdad" (14), "Empleo" (11), "Aborto" (6), "España" (6), y "Derechos civiles" (5). Sin duda, temas más serios y comprometidos y de menor superficialidad política.

\subsubsection{Willy Meyer}

El candidato de Izquierda Plural, Willy Meyer, no solo fue un usuario muy frecuente de Twitter (aunque con el matiz de originalidad señalado más arriba) durante la campaña electoral, también constituyó todo un ejemplo de estrategia combativa, con un lenguaje muy directo dirigido a sus potenciales votantes. Sin embargo, a pesar de estas positivas cualidades en cuanto al uso de Twitter durante la campaña, la mayor parte de sus interacciones estuvieron dirigidas hacia sus propios colegas de partido, con 357 de 489 de las menciones a afiliados o simpatizantes, solo 93 a otros partidos y 39 a otras fuentes diversas, llegando al punto de no establecer ninguna conversación con los ciudadanos ni intentar estimular el debate político.

Es cierto, en cualquier caso, que la cantidad mayor de tuits publicados sí permitieron a Meyer un mayor rango de experimentación, no solo por la cantidad de hashtag utilizados, cuya abundancia también había sido explotada por la cuenta de Elena Valenciano, sino por su tono, mucho más creativo: es el caso de \#puroteatro. Por lo general, el uso de estos hashtag parece improvisado y circunstancial, siendo la excepción el eslogan de campaña \#elpoderdelagente, utilizado como método de protesta por la ausencia de Willy Meyer del debate político televisado por Cañete y Valenciano.

El espíritu combativo de Meyer vuelve a hacer diana en los partidos tradicionales y en la confrontación bipartidista de PP y PSOE, si bien también impregna los temas de interés público tratados en sus tuits, siempre con una patina de la inspiración marxista de su partido. En el caso de "Economía", con 82 tuits en total, el desglose apunta a la "Especulación" (1 1 tuits), el "Fraude" (13), el papel desempeñado por la "Troika" (22) o los "Recortes" en gastos públicos (14); otros temas tanto o más frecuentes fueron "Europa" (112), 
"Empleo" (78), "Derechos civiles" (24), "Servicios públicos" (30) y la "Vivienda" (13).

\subsubsection{Pablo Iglesias}

La irrupción de Pablo Iglesias en la escena política española como líder del nuevo partido Podemos tuvo su gran momento en las Elecciones Europeas de 2014, cuando el partido, con Iglesias a la cabeza, llegó a ocupar cinco escaños. La cantidad de tuits publicados durante el periodo estudiado fue realmente notable, más si tenemos en cuenta la originalidad de sus publicaciones frente a Willy Meyer. Su discurso fue, ante todo, emocional y entusiasta.

Iglesias mencionó a su partido más que cualquier otro candidato, en un total de 177 ocasiones, lo cual refuerza el mensaje de unidad ofrecido por Podemos, pero también vuelve a reincidir sobre la falta de interacción con usuarios fuera de su propio círculo ideológico y de partido. Hay escaso ánimo de interacción en las 27 menciones realizadas al PP y PSOE (de nuevo prevalece la articulación bipartidista del discurso), y las 38 que parecen apuntar fuera, lo hacen, en varias ocasiones a realidades cercanas a Pablo Iglesias y Podemos, como las menciones a los programas @la_tuerka, 8 en total, y @fort_apache, con 4.

El aumento en la cantidad y originalidad de sus tuits viene acompañado de una estrategia mejor planeada, con una frecuencia y variedad de hashtag mayor (fueron utilizados en 112 ocasiones), la mayor parte integrados por el nombre del partido, como \#cartaPodemos, \#ClaroQuePodemos, \#DifundePodemos, \#Podemos1000razones, o \#sinbancospodemos, pero también mediante fórmulas combativas cercanas al estilo utilizado por Meyer para agitar la participación política de los usuarios. Es el caso de \#queteneisqueesconder, un ataque frontal a los métodos para recaudar fondos de PP y PSOE.

La retórica vuelve a prevalecer frente al verdadero debate político, con continuas llamadas al cambio mediante interpelaciones directas a los usuarios. España es el tema capital en el discurso desplegado por Pablo Iglesias con 30 
menciones explícitas, si bien tiende a evitar el nombre mediante expresiones como "este país" o "el país", en las que podríamos interpretar un intento de no causar disconformidad en ciertas sensibilidades nacionalistas. Aunque en un grado menor, con 12 menciones, Europa también está presente, como es natural dado el carácter de estas elecciones. Los temas bisagra fueron conformados por la preocupación por las clases más desfavorecidas ("Pobreza", con 5 tuits; "Desalojos", 5; "Recortes" en el gasto público, 14; "Tercera edad", 7; "Desempleo", 12), la "Casta" (mencionada hasta 17 veces), y los "Bancos" y los casos de "Corrupción", ambos con 12 tuits cada uno.

\subsubsection{UPYD Europa}

Tal y como cabría esperar de la cuenta de un partido político, el estilo del perfil de UPyD para las Elecciones Europeas es más formal, menos personal. El ascenso de UPyD en cuanto a los votos obtenidos podría sugerir, en principio, que la ausencia de la cuenta Twitter de Ricardo Sosa Wagner y de un mayor esfuerzo en el ámbito de la comunicación 2.0 no influyó realmente en las elecciones. Sin embargo, la caída experimentada por UPyD en los meses posteriores a las elecciones hasta su práctica extinción, también pone de manifiesto la oportunidad perdida en cuanto a cumplir su objetivo fundacional y convertirse en la alternativa del bipartidismo.

Debido a esta notable ausencia, la cuenta de UPYD deriva las menciones hacia otros candidatos del partido, subordinados de Sosa, y orienta su mensaje hacia el contenido informativo sobre la campaña y las declaraciones de sus candidatos, e incluso detalles graciosos de las Elecciones.

\section{Conclusiones}

Los discursos políticos estudiados en esta investigación evidenciaron que los políticos españoles no encontraron un encaje adecuado a Twitter dentro de sus campañas políticas durante las Elecciones Europeas. Esto queda patente tras el examen de la explotación de las posibilidades interactivas que los creadores de la herramienta esgrimen habitualmente para defender las bondades de su contribución social. Si hay algo fuera de toda duda después 
de realizar nuestro análisis, es que los principales líderes españoles no emplearon la plataforma de microblogging para interactuar, ni mucho menos frecuentemente, con los ciudadanos durante la campaña de las Elecciones Europeas y en los días posteriores a la celebración de los comicios.

Twitter constituyó durante la campaña y en los días posteriores a la cita electoral un escaparate de información política y declaraciones destinado a cumplir con unos objetivos de comunicación más próximos al paradigma de los medios tradicionales de masas que a una verdadera voluntad de acercamiento a los votantes y una patente interacción con ellos. La escasa familiaridad de la mayoría de partidos estudiados con la herramienta puede ser el resultado involuntario de un fenómeno que, en ocasiones se da con las nuevas tecnologías: la sensación de tener que estar en algún sitio aflora con mucha más urgencia que el conocimiento de cómo estar allí de manera efectiva.

La repetición de eslóganes de campaña y la escasa variedad de hashtags en algunas de las cuentas estudiadas (en especial la que gana las elecciones, lo que redunda en lo defendido por Deltell [2012]) ponen esto especialmente de relieve, por más que nuevas formaciones como Podemos e Izquierda Plural parezcan demostrar un mayor atrevimiento a la hora de probar ciertos recursos -como el propio hashtag- que la herramienta proporciona para estimular el debate.

Sin embargo, no hay pasos más allá, ni siquiera en estas formaciones comunicativamente más innovadoras y con más capacidad de adaptación a las exigencias/posibilidades de la nueva herramienta. Esto es así porque el uso eminentemente informativo de Twitter por parte de los políticos analizados se ve, además, complementado por una marcada tendencia endogámica en el uso de la herramienta: son raras las ocasiones en las que el candidato menciona a usuarios fuera del círculo ideológico conformado por su partido, tanto a sus oponentes como a cuentas sin relación directa con la política. Además, es curioso observar cómo en las menciones, por lo general escasas, que hacen las cuentas estudiadas a otras formaciones políticas, no hay una 
tendencia excesivamente rupturista: todos ellos, incluidos los partidos emergentes, alientan más el bipartidismo que otra cosa con sus menciones.

Los resultados obtenidos en esta investigación también revelan la atención dedicada por los candidatos al lenguaje, más que a la profundidad del mensaje, lo que podría cuestionar la relevancia atribuida a Twitter como generador de debate político o, al menos, que los políticos españoles sepan atraer a los ciudadanos hacia dicho debate y la discusión de temas de interés público. No cabe duda de que las cualidades desplegadas por Internet en general, y Twitter en particular, abren nuevas y verdaderas vías de participación política; pero los resultados obtenidos en esta investigación confirman lo apuntado por otras que la precedieron, pues resulta altamente cuestionable que los políticos usen Twitter con esta finalidad interactiva y que el debate político, en efecto, se esté viendo enriquecido.

\section{Referencias bibliográficas}

ALBAEK, E., VAN DALEN, A., JEBRIL, N., y DE VREESE, C. (2014). Political Journalism in Comparative Perspective. Cambridge: Cambridge University Press.

BERNAL, A. I., y CONGOSTO, M. (2014). Campaña electoral de las elecciones europeas: medios de comunicación vs. viralidad de la Red, http://www.alice-comunicacionpolitica.com/abrir-ponencia. php? $\mathrm{f}=508$ F5422a58d5081411556749-ponencia-1.pdf

BOLLERO, D. (2014). Podemos se consolida como la primera fuerza política en redes sociales, Diario Público, http://www.publico.es/politica/consolidaprimera-fuerza-politica-redes.html

BONETE, F., CEBRIÁN, E., y VÁZQUEZ, T. (2016). Pablo Iglesias' Tweeting Style: Unsuccessfully Aspiring to Renew Spanish Political Discourse. En VVAA, Tweets from the Campaign Trail. Researching Candidates' Use of Twitter During the European Parliamentary Elections. Bruselas: Peter Lang.

BOSCO, A. (2013). Party Change in Southern Europe. Londres: Routledge.

CEBRIÁN, E., y VÁZQUEZ, T. (2013). @marianorajoy VS @conRubalcaba: La campaña electoral de 2011 en las cuentas oficiales de Twitter de los candidatos del PP y del PSOE. En M.R. Pieretti (ed.), Miradas a las pantallas en el bolsillo (pp. 93-108). Madrid: Universidad Camilo José 
Cela,.

CEBRIÁN, E., VÁZQUEZ, T., y OLABARRIETA, A. (2013). ¿̇Participación y democracia en los medios sociales?: El caso de Twitter en las elecciones vascas de 2012, adComunica, 6, 39-63.

DELTELL, L. (2012). Estrategias de comunicación política en las redes sociales durante la campaña electoral del 2011 en España: El caso de eQuo. Jornadas de la Asociación Madrileña de Sociología.

DELTELL, L., CLAES, F., OSTESO, J. M. (2013). Predicción de tendencia política por Twitter: Elecciones Andaluzas 2012, Ámbitos, 22. Recuperado de http://ambitoscomunicacion.com/2013/prediccion-de-tendenciapolitica-por-twitter-elecciones-andaluzas-2012

FERRA, I., y KARATZOGIANNI, A. (2017). A Tale of Cyberconflict in Greece: Polarization and Mobilization for the Greek Referendum on Twitter. En T. STATHOPOULOU, (ed.), Transformations of Protest in Greece. Atenas: Papazisis Publishers \& EKKE. Online available at: https://works.bepress.com/athina_karatzogianni/28/

GEE, J. P. (1999). An Introduction to Discourse Analysis: Theory and Method. Oxon, Routledge.

HOLOTESCU, C., GUTU, D., GROSSECK, G., y BRAN, R. (2011). Micro-blogging meets politics. The influence of communication in 140 characters on Romanian presidential elections in 2009, Romanian Journal of Communication and Public Relations, 13(1), 37-47. Recuperado de https://www.academia.edu/11776357/Microblogging Meets Politics. Th e Influence of Communication in 140 Characters on Romanian Presi dential_Elections in 2009

HOUGHTON D., HAMDAN Z.A., yMARDER B. (2017). Structured Abstract: Political Campaigning on Twitter-The Use of Language, Message Tone, and Implications for Political Marketing Communication from the UK General Election 2015. En M. STIELER (eds), Creating Marketing Magic and Innovative Future Marketing Trends. Developments in Marketing Science: Proceedings of the Academy of Marketing Science. Cham : Springer.

LARSON, A., y MOE, H. (2011). Studying political microblogging: Twitter usersin edmetic, 6(2), 2017, E-ISSN: 2254-0059; pp.185-209. doi: https://doi.org/10.21071/edmetic.v6i2.6929 
the 2010 Swedish election campaign, New Media and Society, vol. 13, n 7. p. 1.180-1.197. Recuperado de http://www.hm.vib.no/files/Larsson_Moe_NM_S_pre_print.pdf

LARSON, A., Y MOE, H. (2013). Representation or participation? Twitter use during the 2011 Danish election campaign, Javnost-The Public, 20(1), 71 88.

Recuperado

de

https://www.researchgate.net/profile/Anders_Larsson6/publication/2601 85602 Representation or Participation Twitter Use During the 2011 Da nish_Election_Campaign/links/Odeec52ff9aff9078e000000.pdf

LILLEKER, D., y JACKSON, N. (2013). Political campaigning, elections and the Internet: Comparing the US, UK, France and Germany. Londres: Routledge

MAMBREY, P., y DÖRR, R. (2011). Local government and social networking technologies in Germany: The example of Twitter. Conference for EDemocracy and Open Government (pp. 249-260). Recuperado de http://www.donau-

uni.ac.at/imperia/md/content/department/gpa/zeg/bilder/cedem/ced em 11 final version.pdf

MICHAILIDOU, A., y TRENZ, H. J. (2010). Mediati(zi)ng EU politics: Online news coverage of the 2009 European Parliamentary elections, Communications, 35(3), 327-346. Recuperado de https://www.researchgate.net/profile/Asimina Michailidou/publication/2 49940043 Mediati(zi)ng EU politics Online news coverage of the 2009 European Parliamentary elections/links/53fafla50cf2e3cbf565e428.pdf SHIRKY, C. (2011). The Political Power of Social Media. Technology, the Public Sphere, and Political Change, Foreign Affairs, 90(1). Recuperado de https://www.foreignaffairs.com/articles/2010-12-20/political-powersocial-media

THE ECONOMIST. (2014). Exit Rubalcaba. The Socialist leader quits after a humiliating election defeat. Recuperado de http://www.economist.com/news/europe/21603040-socialist-leaderquits-after-humiliating-election-defeat-exit-rubalcaba TUMASJAN, A., SPRENGER, T. O., SANDNER, P. G., y WELPE, I. M. (2010). 
Predicting Elections with Twitter: What 140 Characters Reveal about Political Sentiment. Proceedings of the Fourth International AAAI Conference on Weblogs and Social Media, (pp. 178-185). Munich: Technische Universität München. Recuperado de http://www.aaai.org/ocs/index.php/ICWSM/ICWSM10/paper/viewFile/1 441/1852Predicting

VERGEER, M., y HERMANS, L. (2013). Campaigning on Twitter: Microbloggingand Online Social Networking as Campaign Tools in the 2010 General Elections in the Netherlands, Journal of Computer-Mediated Communication, 18, 399-419. Recuperado de http://onlinelibrary.wiley.com/doi/10.1111/jcc4.12023/epdf

VERGEER, M., HERMANS, L., y CUNHA, C. (2012). Web campaigning in the 2009 European Parliament elections: A cross-national comparative analysis, New Media \& Society, 15(1), 128-148. Recuperado de https://www.researchgate.net/publication/258173926_Web_campaignin g_in_the_2009_European_Parliament_elections_A_crossnational comparative analysis

ZUGASTI, R., y PÉREZ, J. (2015). La interacción política en Twitter: el caso de @ppopular y @ahorapodemos durante la campaña para las Elecciones Europeas de 2014, Ámbitos, 28, Recuperado de http://ambitoscomunicacion.com/2015/la-interaccion-politica-entwitter-el-caso-de-ppopular-y-ahorapodemos-durante-la-campanapara-las-elecciones-europeas-de-2014

ZURUTUZA, C. (2012). ¿De qué habla Rubalcaba cuando tuitea? La campaña del candidato socialista en Twitter para las elecciones del $20 \mathrm{~N}$. ponencia presentada para la mesa "Redes Sociales y Campañas Políticas: el uso de Twitter y Facebook por los candidatos". I Congreso Internacional en Comunicación Política y Estrategias de Campaña (Madrid, 6 y 7 de julio).

\section{Cómo citar este artículo:}

Gelado Marcos, Roberto y Bonete Vizcaíno, Fernando (2017). La competencia edmetic, 6(2), 2017, E-ISSN: 2254-0059; pp.185-209. doi: https://doi.org/10.21071/edmetic.v6i2.6929 (c) edmetic, Revista de Educación Mediática y TIC 
digital de los políticos españoles en Twitter. El caso de las elecciones europeas de 2014. EDMETIC, Revista de Educación Mediática y TIC, 6(2), 185-208. doi: https://doi.org/10.21071/edmetic.v6i2.6929 